\title{
Acquisition of the Minor Planet Center code for the Astronomical Observatory of the Technological University of Pereira (W63)
}

\author{
Acquisition of the Minor Planet Center code for the Astronomical Observatory of the \\ Technological University of Pereira (W63)
}

\author{
Santiago Jiménez Villarraga ${ }^{1}$, Edwin Andrés Quintero Salazar. ${ }^{* 2}$, Jairo Alberto Aguirre Galvis. ${ }^{2}$ \\ ${ }^{1}$ Instituto Nacional de Astrofísica, Óptica y Electrónica, INAOE, México \\ ${ }^{2}$ Universidad Politécnica de Madrid, Spain
}

\begin{abstract}
In order to determine the orbits of minor bodies in the Solar System, and in this way foresee collisions with Earth, it is of fundamental importance to perform precise measurement of the relative positions of these objects. The Minor Planet Center (MPC) is a service of the International Astronomical Union (IAU) that receives and distributes the measurements made by observatories all around the world, and likewise uses this information to estimate the orbits of minor bodies. An observatory must firstly demonstrate the capacity to perform measurements with errors below one second of arc through the dutiful application of the MPC methodology before obtaining an international designation. We discuss the methodology implemented at the Astronomical Observatory of the Universidad Tecnológica de Pereira (OAUTP) to obtain the MPC code. In order to do so, we observed the asteroids 1568, 2235 and 713 during the days 6 and 9 of February of 2016 with a 0.4064 m MEADE LX200 GPS telescope, a SBIG ST-2000 XM camera and a f/6.3 focal reducer. The acquired data was sent with an average error of 0.194 arcsec and a standard deviation of $\sigma=0.037 \mathrm{arcsec}$, which granted to the OAUTP the designation W63 under the name Observatorio Astronomico UTP, Pereira. The MPC code obtained certifies the quality of the astrometric data acquired from the OAUTP, opening the gates to the development of observational programs of currently interesting objects, like very Near Earth objects (NEO).
\end{abstract}

Keywords: Asteroids, Astrometry, Data Reduction, Minor Planet Center.

\section{Resumen}

Con el fin de establecer las órbitas de los cuerpos menores del Sistema Solar, y de esta manera anticipar colisiones contra la Tierra, es de fundamental importancia realizar una medición precisa de las posiciones relativas de estos objetos. El Minor Planet Center (MPC) es un servicio de la Unión Astronómica Internacional (IAU) que recibe y distribuye las mediciones realizadas por los observatorios de todo el mundo, utilizando esta información para estimar las órbitas de cuerpos menores del Sistema Solar. Para obtener una designación internacional, un observatorio debe demostrar en primer lugar la capacidad de realizar mediciones con errores por debajo de un segundo de arco a través de la aplicación precisa de la metodología establecida por el MPC. En este artículo se presenta la metodología implementada en el Observatorio Astronómico de la Universidad Tecnológica de Pereira (OAUTP) para obtener el código MPC W63. Para ello, se observaron los asteroides 1568, 2235 y 713 durante los días 6 y 9 de febrero de 2016 con un telescopio GPS MEADE LX200 de 0.4064 m, una cámara SBM ST-2000XM y un reductor focal f/6.3. Los datos obtenidos presentaron un error promedio de 0.194 arcsec y una desviación estándar de $\sigma=0.037$ arcsec, gracias a lo cual el MPC otorgó al OAUTP la denominación W63 bajo el nombre de Observatorio Astronómico UTP, Pereira. El código MPC obtenido certifica la calidad de los datos astrométricos adquiridos desde el OAUTP, abriendo las puertas al desarrollo de programas de observación astrométrica de objetos de interés actual, como por ejemplo los objetos cercanos a la Tierra (NEO).

*Corresponding Author.

E-mail: equintero@utp.edu.co
How to cite: Jimenez, S., Quintero, E., Aguirre, J., Acquisition of the Minor Planet Center code for the Astronomical Observatory of the Technological University of Pereira (W63), TECCIENCIA, Vol. 12 No. 23, 47-56, 2017

DOI: http://dx.doi.org/10.18180/tecciencia.2017.23.6 
Palabras clave: Asteroides, Astrometría, Reducción de Datos, Minor Planet Center (MPC).

\section{Introduction.}

Knowing the precise form of the orbits of asteroids and comets is fundamental for the human being, as it is greatly important to foresee future collisions with Earth [1], and because the understanding of the structure of the orbital space [2] (formed by the chaotic dynamics that gave rise to the Solar System [3]) relies on the precision of the orbital parameters of the bodies that compose it (SSO, by Solar System Objects, [4]). For example, it was believed that the resonance zone 2:1 with Neptune was empty or underpopulated, when in reality the problem was that the bodies 1997 SZ10 and 1996 TR66 had been misclassified as $3: 2$ resonators, actually being $2: 1$ resonators [5] [6]. It could be said then that the orbit determination problem of SSO is important for advancing in the monitoring of their positions to foresee possible impacts with Earth, as well as the importance to study the origin and evolution of the Solar System

However, defining these parameters is essentially a complex task, as for each body a high quantity of observations in a considerable period of time is required, in a way that the uncertainty of the orbit becomes small [7]. Characterizing the orbits of SSO's becomes therefore a fundamentally collaborative task, in which the participation of observatories around the world providing this information is mandatory, with enough quality to obtain convergence in the algorithms of orbit determination. This is a reason the Minor Planet Center (MPC) was created, a service of the International Astronomical Union (IAU), whose purpose is to receive and distribute the position data delivered by the observatories around the world that have the MPC code. It is also a function of the MPC the identification, designation and calculation of the orbital parameters of these bodies ${ }^{2}$.

In order to have a successful process of orbits determination, it is necessary to use high precision data. Therefore, the MPC requires that the observatories reporting astrometric data meet a set of minimum quality standards. Specifically, they grant designations to the observation sites which demonstrate the ability to deliver consistently data with errors below one arcsec. This undoubtedly establishes an important filter that is not easy to meet, given that in order to accomplish this requirement it is necessary at first place to count with the appropriate equipment, that besides a robotic telescope, includes a CCD camera and the appropriate software to perform the data reduction. In second place, it is necessary to develop the correct methodology to successfully accomplish the process, as in this area, in which such precise measurements are imperative, it is common to make mistakes that could seriously affect the quality of the obtained information.

In spite of the international efforts, and the huge advances made in the number of bodies discovered, there is s till an important population of potentially hazardous bodies for Earth that have not yet been found. This is depicted for example in the Chelyabinsk event which happened without previous knowledge [8]. Moreover, the idea that NASA would not be capable to accomplish the purpose of discovering at least $90 \%$ of the minor bodies with diameters over $140 \mathrm{~m}$ by the year 2020 has recently come up [9], which makes combining efforts from all over the world even more relevant. In Colombia, there are very few works on this direction, such as the one by the Observatory of the Nariño University (which has an MPC code). The purpose of this document consist in describing and discussing the asteroids astrometry program of the Astronomical Observatory of the Technological University of Pereira (OAUTP). The careful work carried out resulted on the designation of the MPC code W63 for the OAUTP whose name appears as Observatorio Astronomico UTP, Pereira. We aim to produce a useful reference for other observatories with similar objectives and motivate the establishment of collaborations in this subject.

\section{Materials and Methods.}

For obtaining the observatory code it is necessary to follow the procedure requested by the MPC. We first make two clarifications about the procedure shown in the flux diagram in the Fig. 1. As it is pointed out in the figure, the MPC requires observations of two different nights. In principle the nights are requested to be close enough, but if the weather prevents it, they can be separated by up to a few weeks. In the flux diagram a maximum separation of a month has been set. Likewise, the MPC requests that each of the $\mathrm{n}$ bodies is observed at least twice within an hour. It is not valid to observe a body just once per night. Furthermore, each observation must have a residue equal or lower than one arcsec. If these conditions are met, the observing run is considered as a successful one. As it will be shown further ahead, in this work three asteroids were picked out for observation. In the following, the procedure to manage measurements with errors under the arcsec detailed.

The astrometry of asteroids can be understood as a three stages procedure: planning, observation and data reduction. The particulars of each stage will be described below within the context of the OAUTP. 


\section{TECCIENCIA}

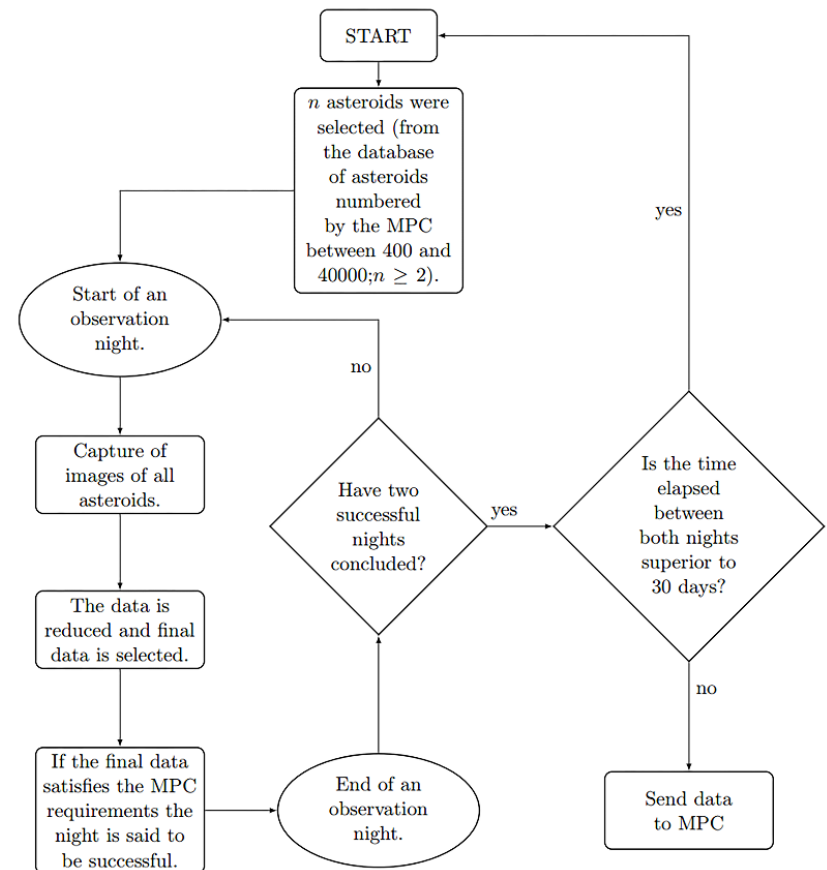

Figure 1 Flux diagram of the required procedure to obtain an MPC code. In this particular case three asteroids were selected, i.e., $n$.

\subsection{Observation planning}

The planning consists in building a list of the objects of interest or places in the celestial sphere to be observed. As in this case the interest was to obtain an MPC code, the guidelines in the Guide to Minor Planet Astrometry [10] were followed. Specifically, three different bodies with different magnitudes were selected. It is advisable that when starting the project the selected asteroids are located east in order to guarantee their observability during sufficient time. It is also advisable to select bodies located within a field of view with a high population of stars, as it will be shown ahead, the process of reduction is more precise with a greater number of reference stars. The selection was made employing the software Toolkit For CCD Astrometry® from the project CLEA ${ }^{1}$. This software allows to filter asteroids from the MPC database that meets certain searching criteria. Usually they are filtered by places in the celestial sphere and magnitudes. The selected asteroids for this project were (713) Luscinia of apparent magnitude for the date of $\approx$ $14.5 \mathrm{~V},(2235)$ Vittore (magnitude $\approx 14.8 \mathrm{~V}$ ) and $(1568$ ) Aisleen (magnitude $\approx 15.4 \mathrm{~V}$ ).

\subsection{Observations}

The Astronomical Observatory of the Universidad Tecnológica de Pereira counts with a MEADE® LX200
GPS telescope of $40.64 \mathrm{~cm}$. It is employed with a SBIG ST 2000 XM CCD camera and an f/6.3 focal reducer ${ }^{2}$. Before each observation night, it is necessary to ensure that the computer's clock in which the photographies will be taken, is synchronized with some internet standard time server ${ }^{3}$. This guarantees that the registered time at the image header matches the real time. Besides, before starting acquiring images, it is necessary to verify that the field of view is the proper one. For this purpose the software SAOImage DS9® ${ }^{4}$ was employed. This allows to search fields of the desired size centered in a specific set of coordinates, taken from the catalog ESO-DSS I/II. For example, in the Fig. 2 a comparison between one of the observed fields with the field obtained from the catalogue is shown. Once verified the field, the process can be carried on. In this case, the data was taken on the 6 and 9 of February of 2016, with three different observations per asteroid, per observing night, as the MPC demands [10].

\subsection{Data Reduction}

It is necessary to proceed to the data processing of each image. This implies knowing the coordinates in pixels of the stars and asteroids displayed in the field. For this, we should bear in mind that they are punctual light sources and their intensities can be modeled through a PSF (point-spread function), which is usually fitted as a Gaussian curve. This allows the estimation of the centroids for each body in the image. For example, the Fig. 3 shows the level curve for the PSF of one of the images of the asteroid 2235. A parameter associated with the PSF is the Signal to Noise Ratio (SNR). A low SNR implies out that the body's PSF cannot be softly adjusted and, therefore, the estimation of the centroid might produce important errors. Each astronomical observatory must pay attention to this fact and establish criteria of their own to control the quality of their data. After an analysis of the obtained images, and based on the instrumentation and environmental conditions of the observatory at which the measurements are made, in this work the value of an asteroid's position was considered useful if fulfilling the condition: $\mathrm{SNR} \geq 11$.

With this information of the coordinates in pixels of the image, the coordinate's transformation of Right Ascension and Declination are computed $(\alpha, \delta)$ in the celestial sphere through a LSPC process (least-squares plate constants [11]), 


\section{TECCIENCLA}

for which it is necessary that in each image's field a good amount of stars are present, so they can be compared with a reference catalog. This process is automatically performed via the software Astrometrica ${ }^{\circledR}$ [12]. It is necessary to be very careful when the identification of the studied body is made, as according to reports from the MPC, one of the most common sources of mistakes by the observers are bodies incorrectly identified. For this reason the functions blink or stack and track from Astrometrica ${ }^{\circledR}$ are used to identify the body that is moving using each sequence of photos taken for every asteroid. In the Fig. 4 the stacked images for the asteroids 713 and 1568 are shown, meanwhile in Fig. 5 asteroid 2235 is encircled in the displayed image. Once the asteroids are identified their positions are calculated. Finally it is very important to notice that we used the UCAC-4 catalog as stellar reference, as suggested by the MPC [10].

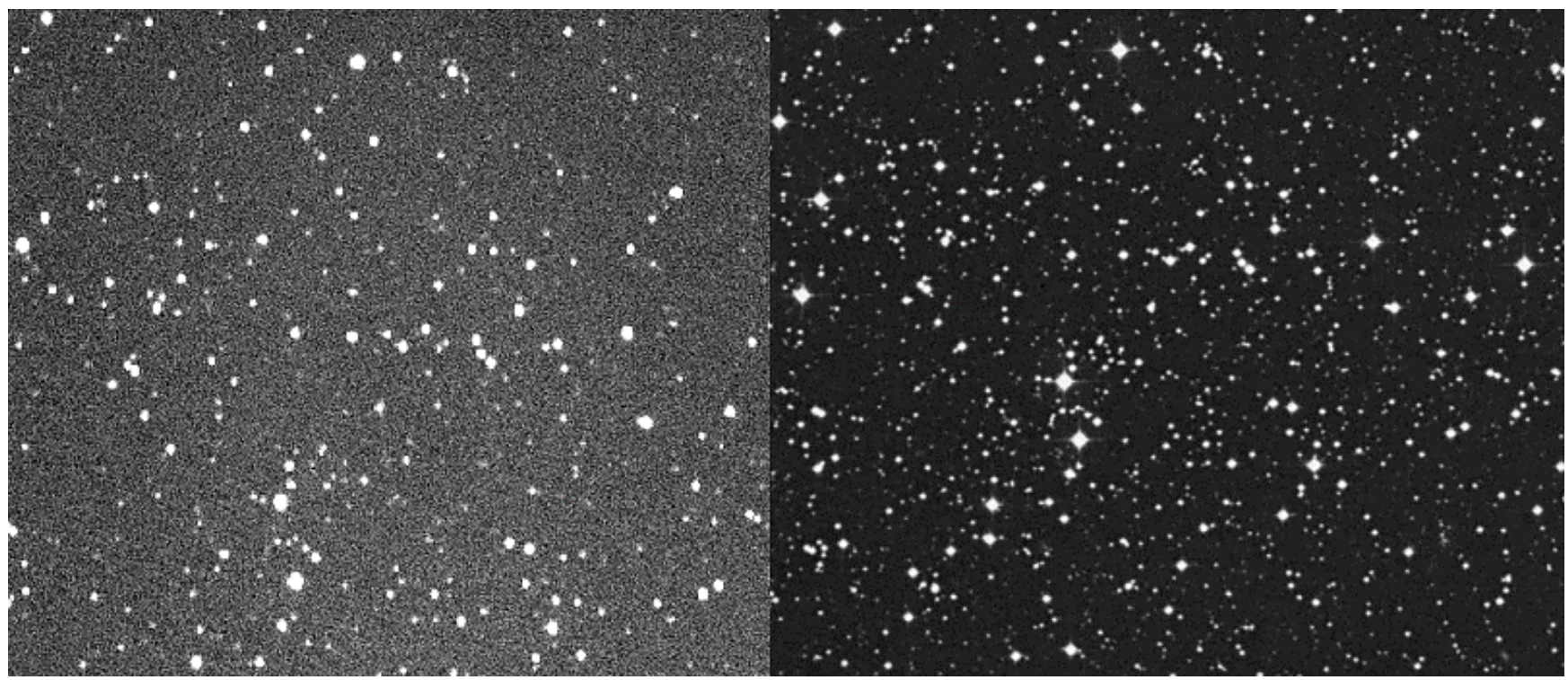

Figure 2 Comparison between the obtained field and the field from the catalog ESO-DSS I/II.

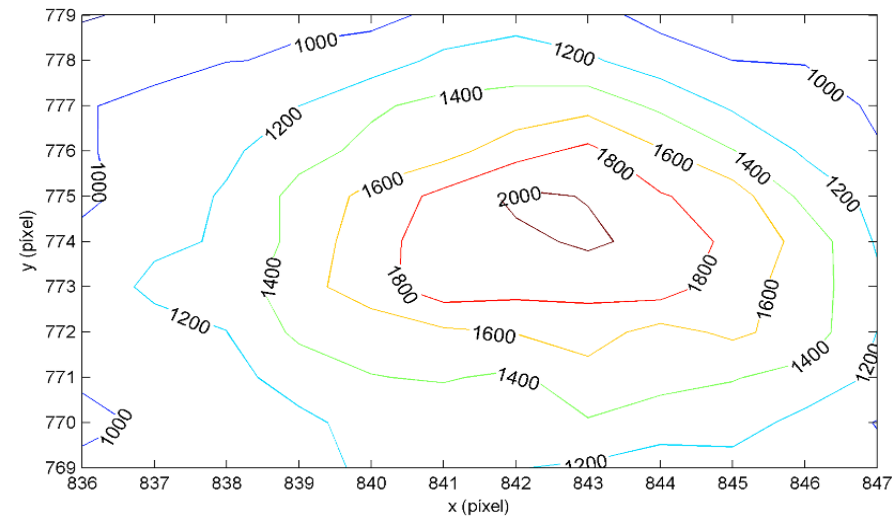

Figure 3 Level curves for the PSF of one of the images taken of the asteroid 2235 Vittore. Each curve indicates the intensity values registered in Analog Digital Units (ADU's). Given that the images were captured with a camera that has an analog to digital conversion of 16 bits in depth, the ADU's range is from 0 to 65535 . 


\section{TECCIENCIA}

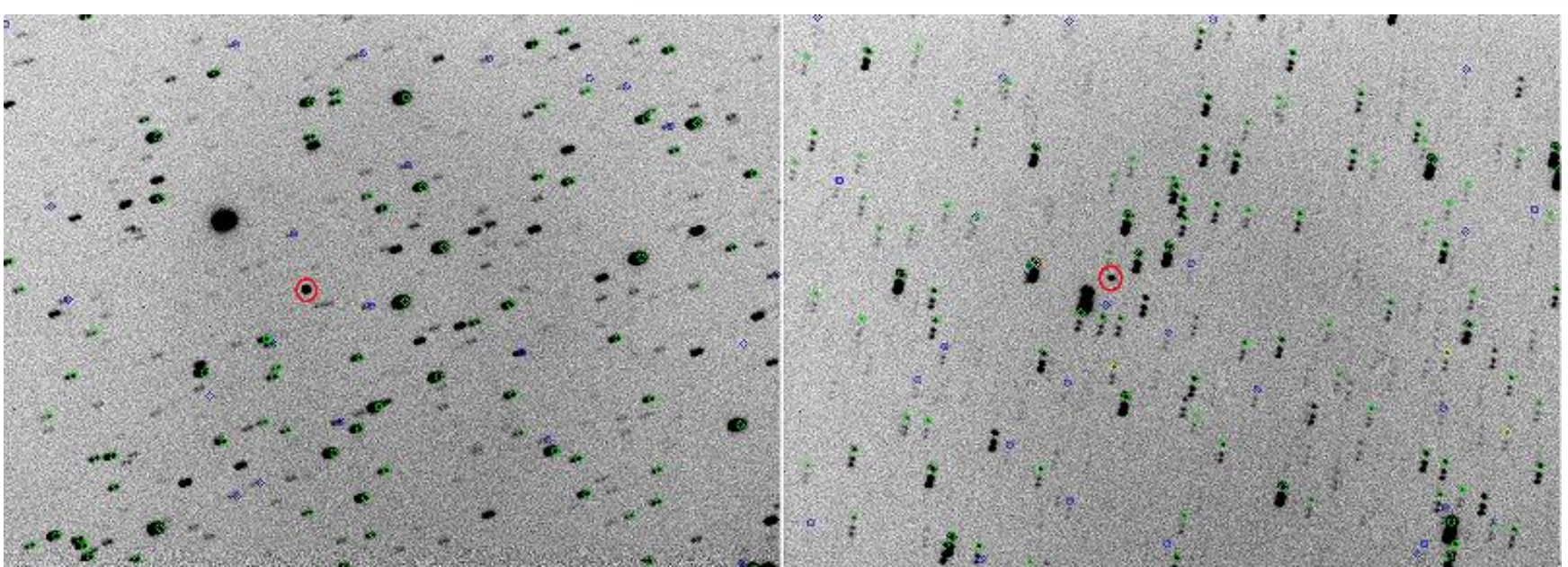

Figure 4 Stack of images. In the left figure the asteroid 713 is encircled in red and in the image on the right the asteroid 1568 is highlighted. The field of view is 33 × 25 arcmin. The Green circles represent the reference stars identified by Astrometrica in the photography and used for calculation of asteroids position. The blue circles represent objects within the image that are not recognized by Astrometrica (possibly noise). the

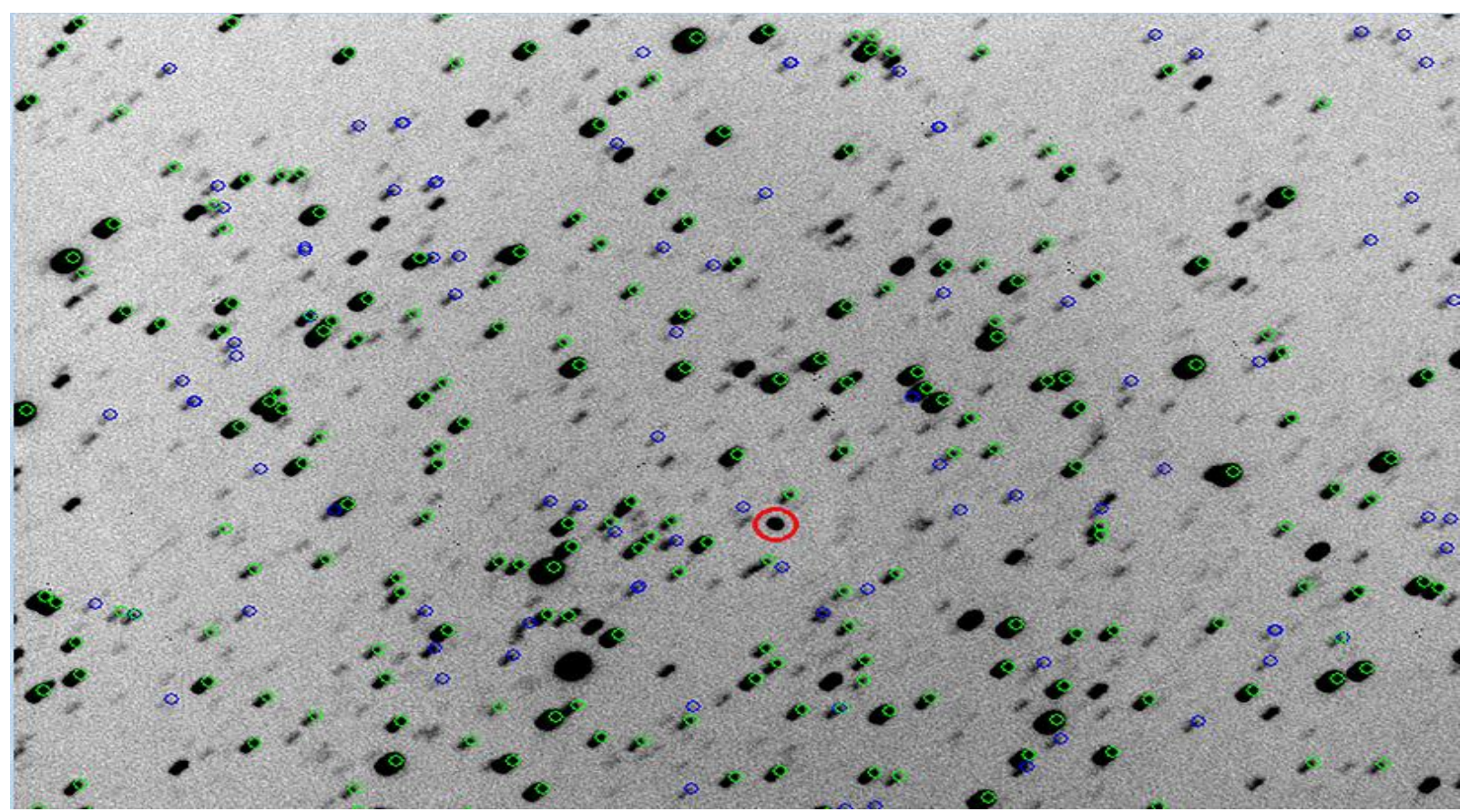

Figure 5 Stack of images of the asteroid 2235. The field of view is $33 \times 25 \operatorname{arcmin}$. The Green circles represent the reference stars identified by Astrometrica in the photography and used for calculation of the asteroids position. The blue circles represent objects within the image that are not recognized by Astrometrica (possibly noise). 


\section{TECCIENCIA}

\section{Result}

As it was decided to use a sample comprising three asteroids to obtain the MPC code and collect three observations per asteroid per observing night, a total number of 18 measurements were sent to the MPC. However, more data for these celestial bodies was taken in such a way that it enabled to check the quality of the results.

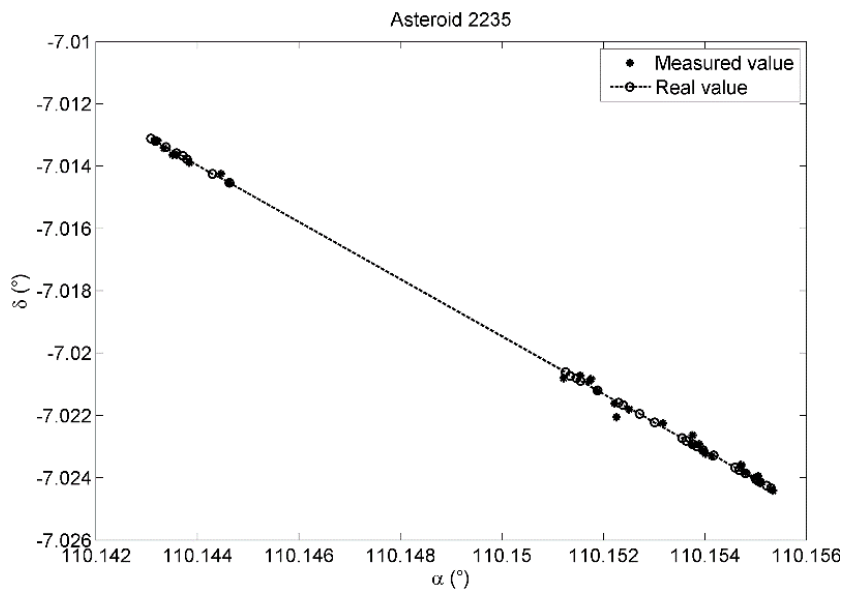

Figure 6 Comparison between the measured positions and

Considering the positions delivered by the JPL as exact values, the errors of the measurements in this work were calculated. In the Fig. 7 the obtained errors are shown in each axis for all the observations obtained for the three asteroids chosen as sample and in the Fig. 8 the total error was calculated as:

$$
=\sqrt{\epsilon_{\alpha}^{2}+\epsilon_{\delta}^{2}}
$$
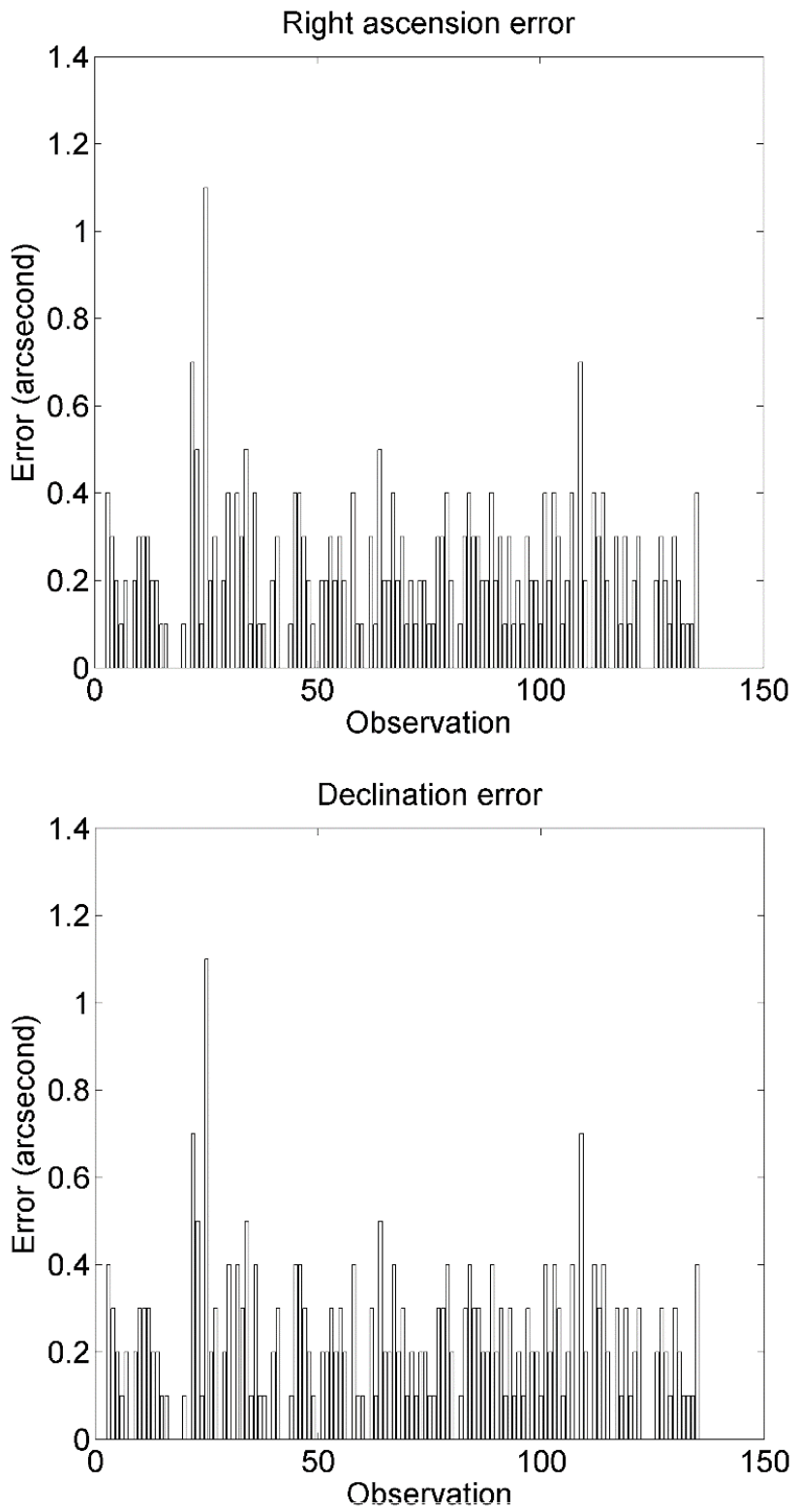

Figure 7 Errors obtained in each coordinate for all observations carried out.

Where $\varepsilon \alpha$ is the error in Right Ascension and $\varepsilon \delta$ in Declination. It is noticed that $97.037 \%$ of the results show errors below 1 arcsec and over $80 \%$ of them are inside a range of 0.6 arcsec. This is important given that the MPC considers useful data with errors below 1 arcsec. After considering the errors in the measurements 18 observations were finally chosen to be sent to the MPC. The requirement of the MPC to send only 2 or 3 observations (in this case 3 


\section{TECCIENCLA}

were chosen) per observing night, for each chosen body (cover a time range of approximately an hour), was entirely fulfilled. Secondly, the preference of the MPC for the consistency of the data was also taken into account. The results sent had an average error of 0.194 arcsec and standard deviation of $\sigma=0.037$ arcsec. The information was sent as ASCII files as it is shown in the Fig. 9. Finally, a week later, the designation W63 was received from the MPC [13].

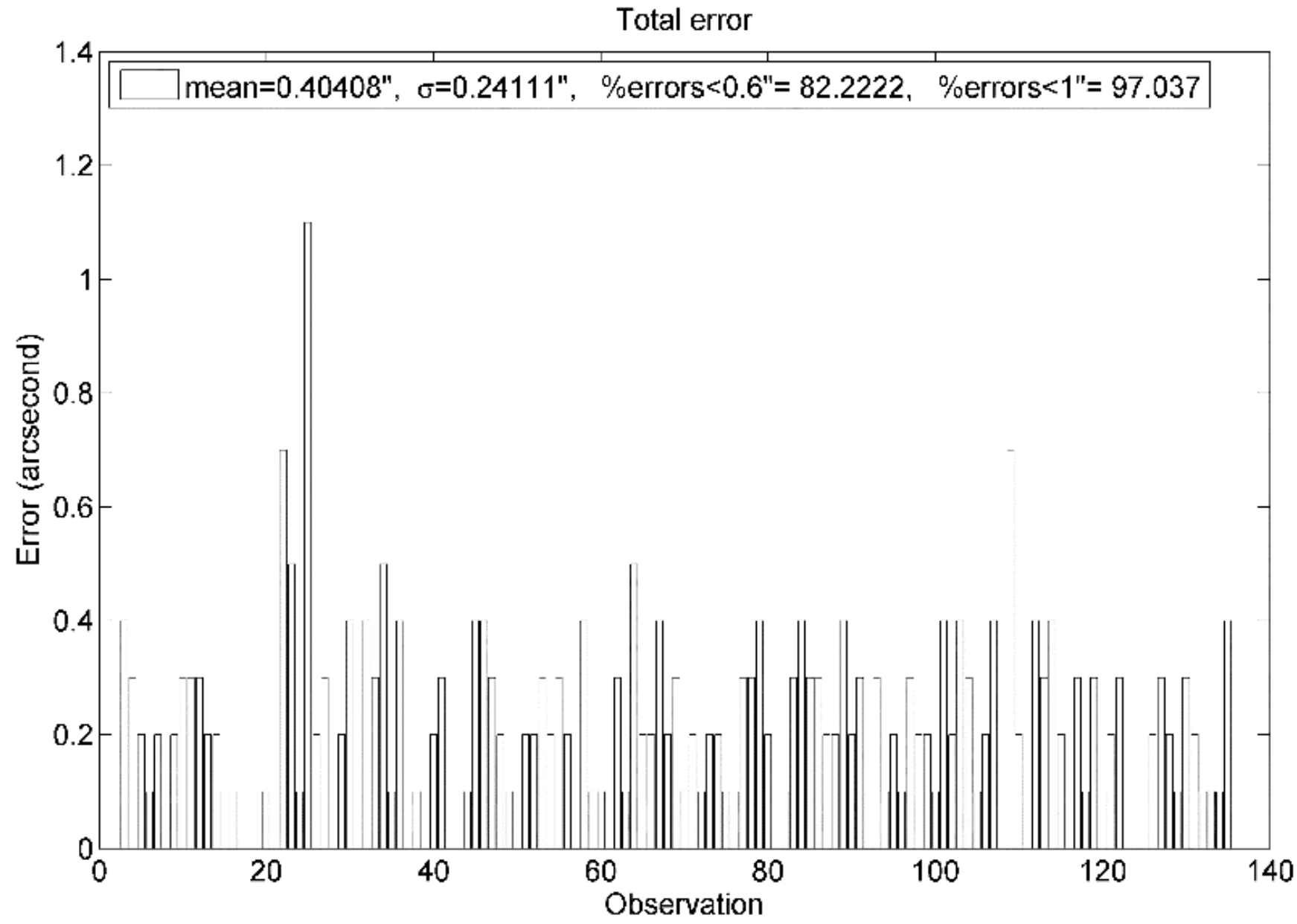

Figure 8 Total Error obtained for all the acquired observations. 


\section{TECCIENCIA}

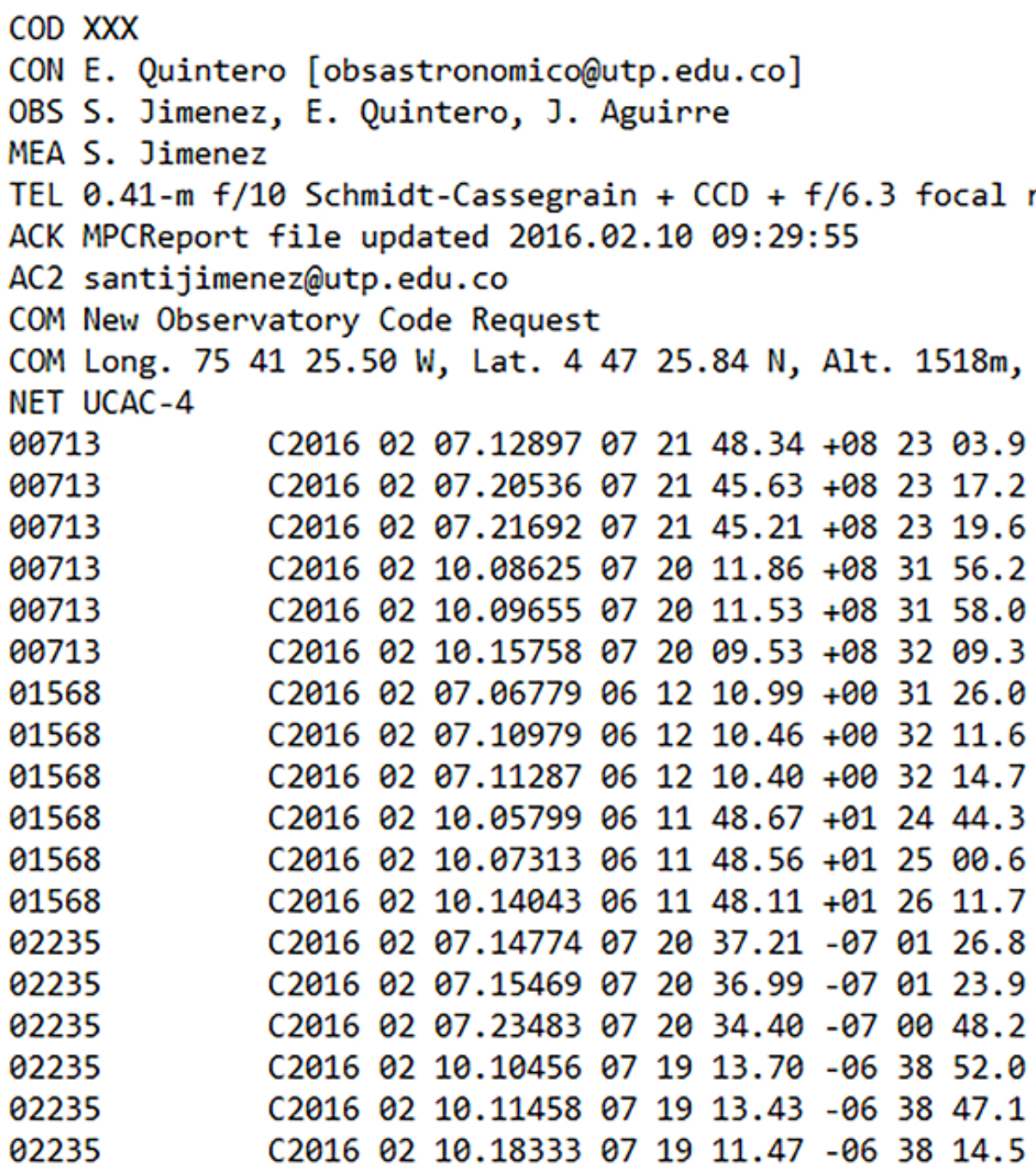

\section{Google Earth}

$\begin{array}{ll}14.7 \mathrm{~V} & X X X \\ 14.5 \mathrm{~V} & X X X \\ 14.4 \mathrm{~V} & X X X \\ 14.5 \mathrm{~V} & X X X \\ 14.5 \mathrm{~V} & X X X \\ 14.5 \mathrm{~V} & X X X \\ 15.1 \mathrm{~V} & X X X \\ 15.3 \mathrm{~V} & X X X \\ 15.3 \mathrm{~V} & X X X \\ 15.4 \mathrm{~V} & X X X \\ 15.4 \mathrm{~V} & X X X \\ 15.2 \mathrm{~V} & X X X \\ 14.6 \mathrm{~V} & X X X \\ 14.6 \mathrm{~V} & X X X \\ 14.7 \mathrm{~V} & X X X \\ 14.8 \mathrm{~V} & X X X \\ 14.8 \mathrm{~V} & X X X \\ 14.9 \mathrm{~V} & X X X\end{array}$

Figure 9 Data sent to the MPC in ASCII format of 80 columns.

\section{Conclusions}

We discussed the program of astrometry of asteroids at the Astronomical Observatory of the Universidad Tecnológica de Pereira in Colombia. Descriptions of the instruments and methods to obtain the MPC code have been made accordingly. We also pointed out the essential elements in the process to make astrometry and the those requiring more attention have been emphasized. As it was shown, the OAUTP has the instrumental capability to send astrometric data with errors well below the requirements of the MPC.

As future work we proposed to study the instrumental limits at the OAUTP, and manage the application of observational techniques that have allowed other observatories to $\mathrm{m} 4$. D easure very faint objects with similar equipment (for example the stack and blink of a great amount of photographies of the same object, or the implementation of guiding strategies with guide cameras to achieve long exposure photographies). The importance of this resides in the fact that asteroids very close to Earth, known as NEO asteroids, are the ones occupying the attention of the MPC, as their discovery and characterization is fundamental to anticipate possible collisions with our planet in the future. These bodies represent an important challenge given their low brightness and high daily motion.

The errors presented in Fig. 8 are attributed to various causes, among which stand out: imperfections in the guiding system of the telescope (given that the guiding camera was not used), seeing (which for the OAUTP is averaged to 2.2 arcsec) and deficiencies in focus (which is adjusted manually from the PSF shape observation). However, the 


\section{TECCIENCLA}

errors obtained for the measured asteroids positions are located below 1 arcsec (the $82.2 \%$ is located below 0.6 arcsec), achieving the requested measurements by the MPC. Even so, future work has been planned with the adaptation of a guide camera for telescope autoguiding, using optic adaptative equipments to diminish the adverse effects caused by seeing and the implementation of software that allows a fine automatic adjustment of focus.

Additionally, the characterization of the errors obtained by the OAUTP will be carried out as a future work, with the intention to estimate a distribution function for these. In the orbit determination, especially with the kind and quantity of modern data, statistics play an important role nowadays [14]. This is truth for several reasons. For example, the case described in the introduction, in which two bodies were classified incorrectly could have been avoided if the orbital parameters were known with confidence intervals; and for this it is necessary to know the kind of errors in the data used to produce these parameters. Moreover, statistics itself can be useful as a method to determine orbits [15].

\section{References}

[1] D. Morrison, "The Spaceguard Survey: Report of the NASA International Near-Earth-Object Detection Workshop", Moffett Field, CA, 1992.

[2] A. Milani and G. Gronchi, Theory of Orbit Determination. Cambridge University Press, 2010.

[3] A. Morbidelli, Modern Celestial Mechanics: Aspects of Solar System Dynamics, vol. 1. Taylor \& Francis, 2002.

[4] A. Milani, G. F. Gronchi, and Z. Knežević, "New Definition of Discovery for Solar System Objects", Earth. Moon. Planets, vol. 100, no. 1-2, pp. 83-116, Mar. 2007.

[5] G. Bernstein and B. Khushalani, "Orbit Fitting and Uncertainties for Kuiper Belt Objects", Astron. J., vol. 120, no. 6, p. 3323 , 2000

[6] H. D. G. Rodriguez, E. A. Q. Salazar y L. F. Cardona, «Development of a Magnetic Loop Antenna for the Detection of Jovian Radiowaves at $20.1 \mathrm{MHz}, »$ TECCIENCIA, vol. 11, n 20, pp. 41 46, 2016.

[7] J. Danby, Fundamentals of Celestial Mechanics, 2nd ed. Richmond: Willman-Bell, 1992.

[8] J. I. Zuluaga and I. Ferrin, "A preliminary Reconstruction of the Orbit of the Chelyabinsk Meteoroid", arXiv Prepr. arXiv1302.5377, 2013.

[9] D. Oberhaus, "The Underfunded, Disorganized Plan to Save Earth from the Next Giant Asteroid," 2015. [Online]. Available: https://motherboard.vice.com/en_us/article/the-underfunded-disorganizedplan-to-save-earth-from-the-next-giant-asteroid. [Accessed: 15-Feb-2016] [10] Minor Planet Center, "Guide to Minor Body Astrometry", 2015. [Online]. Available: http://www.minorplanetcenter.net/iau/info/Astrometry.html. [Accessed: 20-Feb-2016].

[11] J. Kovalevsky and P. K. Seidelmann, Fundamentals of Astrometry. Cambridge University Press, 2004.

[12] H. Raab, "Detecting and Measurin[1] D. Morrison, "The Spaceguard Survey: Report of the NASA International Near-Earth-Object Detection Workshop", Moffett Field, CA, 1992.

[12] A. Milani and G. Gronchi, Theory of Orbit Determination. Cambridge University Press, 2010.
[13] A. Morbidelli, Modern Celestial Mechanics: Aspects of Solar System Dynamics, vol. 1. Taylor \& Francis, 2002.

[14] A. Milani, G. F. Gronchi, and Z. Knežević, "New Definition of Discovery for Solar System Objects", Earth. Moon. Planets, vol. 100, no. 1-2, pp. 83-116, Mar. 2007.

[5] G. Bernstein and B. Khushalani, "Orbit Fitting and Uncertainties for Kuiper Belt Objects”, Astron. J., vol. 120, no. 6, p. 3323 , 2000

[6] H. D. G. Rodriguez, E. A. Q. Salazar y L. F. Cardona, «Development of a Magnetic Loop Antenna for the Detection of Jovian Radiowaves at $20.1 \mathrm{MHz}$,» TECCIENCIA, vol. 11, nº 20, pp. 41 46, 2016.

[7] J. Danby, Fundamentals of Celestial Mechanics, 2nd ed. Richmond: Willman-Bell, 1992.

[8] J. I. Zuluaga and I. Ferrin, "A preliminary Reconstruction of the Orbit of the Chelyabinsk Meteoroid", arXiv Prepr. arXiv1302.5377, 2013.

[9] D. Oberhaus, "The Underfunded, Disorganized Plan to Save Earth from the Next Giant Asteroid," 2015. [Online]. Available: https://motherboard.vice.com/en_us/article/the-underfunded-disorganizedplan-to-save-earth-from-the-next-giant-asteroid. [Accessed: 15-Feb-2016]. [10] Minor Planet Center, "Guide to Minor Body Astrometry", 2015. [Online]. Available: http://www.minorplanetcenter.net/iau/info/Astrometry.html. [Accessed: 20-Feb-2016].

[11] J. Kovalevsky and P. K. Seidelmann, Fundamentals of Astrometry. Cambridge University Press, 2004.

[12] H. Raab, "Detecting and Measuring Faint Point Sources with a CCD", in Proceedings of the Meeting on Asteroid and Comets in Europe, 2003, p. 4.

[13] Minor Planet Center, "The Minor Planet Circulars/Minor Planets and Comets M.P.C. 97571”, 2016.

[14] M. Carpino, A. Milani, and S. R. Chesley, "Error Statistics of Asteroid Optical Astrometric Observations", Icarus, vol. 166, no. 2, pp. 248-270, Dec. 2003.

[15] K. Muinonen, "Orbital Covariance Eigenproblem for Asteroids and Comets", Mon. Not. R. Astron. Soc., vol. 280, no. 4, pp. 1235-1238, Jun. 1996.

g Faint Point Sources with a CCD”, in Proceedings of the Meeting on Asteroid and Comets in Europe, 2003, p. 4.

[13] Minor Planet Center, "The Minor Planet Circulars/Minor Planets and Comets M.P.C. 97571”, 2016.

[14] M. Carpino, A. Milani, and S. R. Chesley, "Error Statistics of Asteroid Optical Astrometric Observations", Icarus, vol. 166, no. 2, pp 248-270, Dec. 2003.

[15] K. Muinonen, "Orbital Covariance Eigenproblem for Asteroids and Comets", Mon. Not. R. Astron. Soc., vol. 280, no. 4, pp. 1235-1238, Jun. 1996. 